\title{
EXPLOTACIÓN DE FRUTOS DE ALGARROBO (PROSOPIS SPP.) POR GRUPOS CAZADORES RECOLECTORES DEL NORESTE DE PATAGONIA
}

\author{
EXPLOITATION OF ALGARROBO (PROSOPIS SPP.) FRUITS BY \\ HUNTER-GATHERERS FROM NORTHEAST PATAGONIA
}

\author{
Aylen Capparelli ${ }^{1}$ y Luciano Prates ${ }^{1}$
}

\begin{abstract}
La importancia de los alimentos vegetales en la subsistencia de los cazadores-recolectores del extremo sur de América del Sur ha sido desigualmente tratada en los estudios arqueológicos. Aunque son muy abundantes las referencias etnohistóricas sobre su uso entre los indígenas del área, las pruebas arqueobotánicas son aún escasas. En este trabajo se analizan los macrorrestos de frutos de Prosopis spp. (algarrobo) de un sitio del norte de Patagonia, Argentina. La muestra analizada está constituida por 77 partes de vaina procedentes del sitio Angostura 1, ubicado en el valle medio del río Negro, cuya cronología es de ca. 900 años a.p. Las partes de vainas y sus patrones de fragmentación fueron comparados con resultados obtenidos a partir de diseños experimentales basados en datos etnohistóricos. Sobre la base de la información presentada se plantea que en el sitio las vainas fueron procesadas para consumir tostadas. La subrepresentación de macrorrestos que avalen el uso de Prosopis en Patagonia podría deberse a una baja visibilidad arqueológica del consumo de tipo directo de este recurso; en el presente caso se observa que la visibilidad aumenta cuando nuevas prácticas de procesamiento poscosecha son incorporadas entre los eventos de recolección y consumo.
\end{abstract}

Palabras claves: cazadores-recolectores del extremo sur de Sudamérica, Holoceno Tardío, consumo de frutos de Prosopis.

The significance of edible plants in the subsistence of the hunter-gatherers of Southern South America has not been dealt with in depth by archaeological research. Although there are many ethnohistorical references about plant consumption by local indigenous people, archaeobotanical evidence of this practice is still scarce. In this paper, fruit macroremains of Prosopis spp. (algarrobo) coming from an archaeological context of Northern Patagonia (Argentina) are analized. The sample is comprised of 77 charred parts of Prosopis pods from the site Angostura 1 (middle Río Negro valley), with an age of ca. 90014 C yrs. BP. Pod parts and fragmentation patterns were compared with those of experimental ess ays designed on the basis of ethnohistorical data. We propose that Prosopis pods from this site were roasted before being eaten. The underrepresentation of edible Prosopis macroremains in Patagonia may be due to the low visibility of direct consumption of wild plants in the archaeological record; in the present case it is observed that this visibility increases when post-harvesting practices were incorporated between gathering and consumption stages.

Key words: Southern South America hunter-gatherers, late Holocene, Prosopis fruit consumption.

Las plantas leguminosas del género Prosopis revisten singular importancia arqueológica debido a que han sido explotadas por los seres humanos de manera sistemática y con diversos fines a lo largo del mundo y a través del tiempo (Capparelli 2007). Se registraron evidencias de su uso en EE.UU., México, Perú, Bolivia, Chile, Paraguay, Argentina, India y Sudeste del Levante, desde el paleolítico superior en el Viejo Mundo (Mc Laren y Capparelli 2004) y el período Arcaico en el Nuevo Mundo (Fernández Distel 1989), hasta la actualidad. Si bien el género posee una distribución espacial relativamente amplia, que incluye el Viejo Mundo -cercano oriente y Norte de África- y buena parte del continente americano -desde el sudoeste de Norteamérica hasta el Cono sur de Sudamérica- la mayor parte de las 44 especies conocidas se encuentra en Sudamérica $(\mathrm{n}=32)$ (Figura 1, Tabla 1); ocho son nativas de Norteamérica y solo cuatro del Viejo Mundo. A pesar de que la taxonomía de este género está siendo revisada y simplificada en algunos países de Sudamérica (p.ej., Perú, Beresford-Jones 2011), en Argentina aún está vigente la clasificación de Burkart (1940, 1952, 1976, Tabla 1), quien reconoce 28 especies nativas y considera a este territorio como el centro de origen primario del género. La Patagonia constituye su confín de distribución más austral.

1 CONICET, División Arqueología, Museo de La Plata, Universidad Nacional de La Plata, Paseo del Bosque s/n CP 1900, La Plata, Argentina. aylencapparelli@gmail.com; lprates@fcnym.unlp.edu.ar 


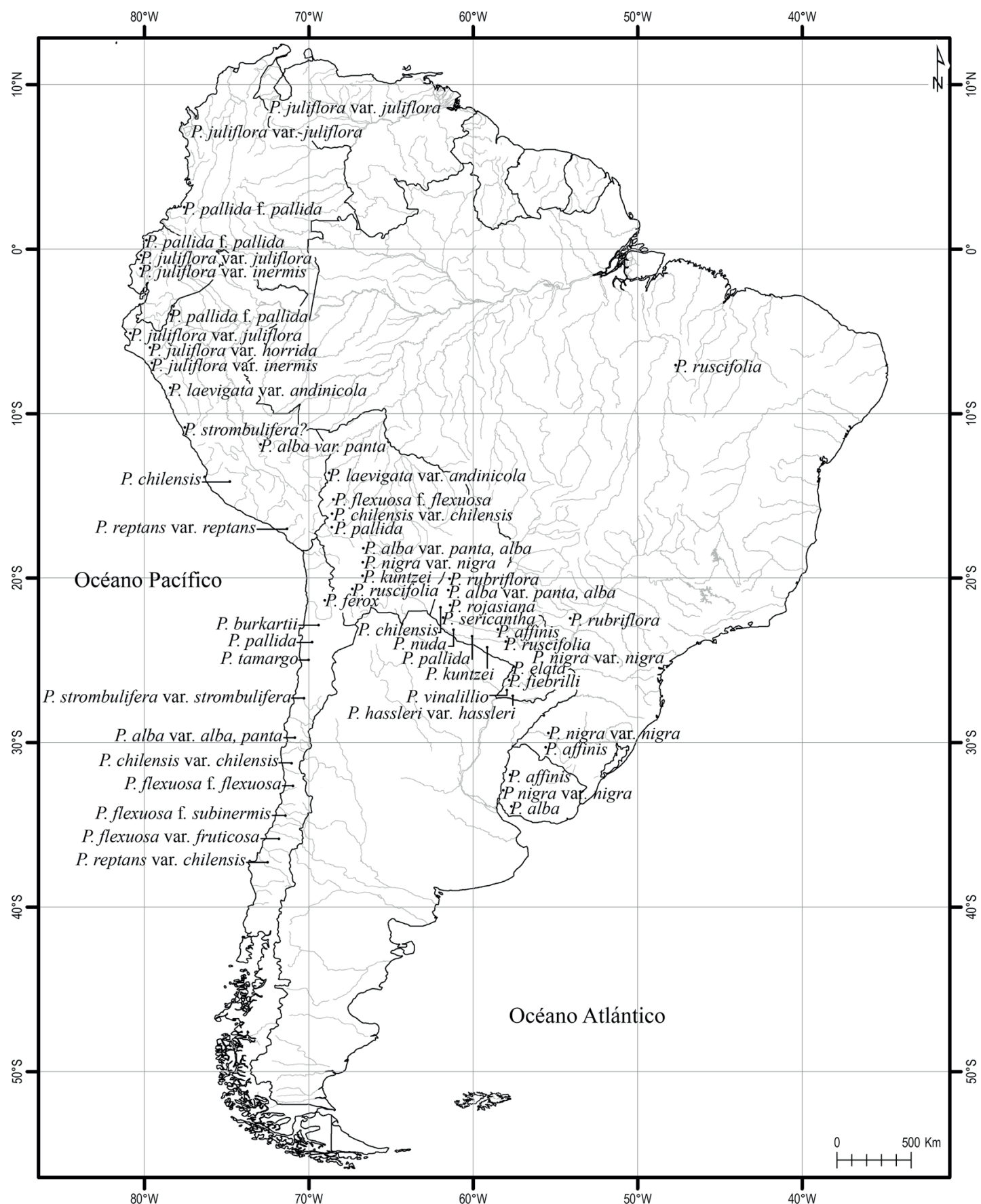

Figura 1. Distribución de especies del género Prosopis en Sudamérica (excepto Argentina). Distribution of Prosopis in South America (with exception of Argentina). 


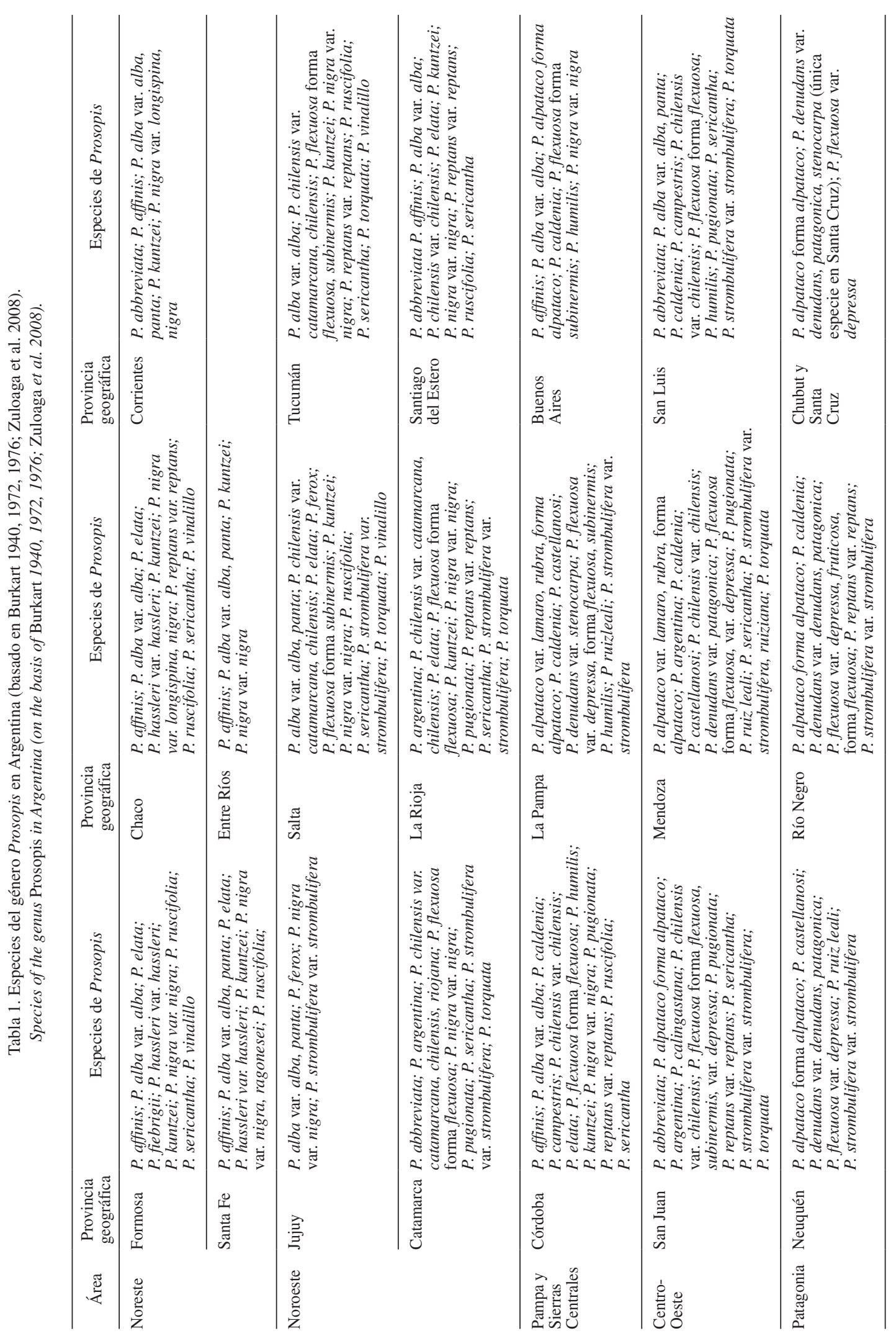


La localización de los registros del uso alimentario de Prosopis es coherente con la dispersión geográfica del género. Mediante fuentes etnográficas y etnohistóricas fue documentado el consumo de frutos de 16 de las 44 especies conocidas: once en Sudamérica, cuatro en Norteamérica y una en el Viejo Mundo (compilado en Capparelli 2007). Entre las especies sudamericanas tradicionalmente explotadas se encuentran: P. chilensis (Molina) Stuntz emend. Burkart, P. pallida (Humboldt \& Bonpland ex Willdenow) H.B.K. (Beresford-Jones 2011; Felker 1979; Latcham 1936; Horkheimer 2004) -en Chile, Perú, Bolivia y Paraguay-, P. alba Grisebach (Villagrán et al. 1998) -en Chile y en Argentina-, y P. nigra (Grisebach) Hieronymus, $P$. flexuosa DC, $P$. chilensis, $P$. ruscifolia Grisebach, $P$. vinalillo Stuckert, $P$. elata (Burkart) Burkart, $P$. torquata (Cavanilles. ex Lagasca) DC, P. hassleri Harms., y P. ferox Gris (Capparelli 2007) -en Argentina-. En Argentina Prosopis fue utilizado tanto por grupos con una economía básicamente agrícola (i.e. diaguitas), como por aquellos que combinan caza con recolección y, eventualmente, pesca y horticultura (i.e. huarpes, tobas, wichís, pilagás, mocovíes, abipones, guaraníes, chanátimbú, pampas, puelches, mapuches y tehuelches) (Capparelli 2008 y trabajos allí citados). En todos los casos, las vainas constituían un recurso alimentario importante en los meses de maduración, tanto para su consumo fresco como para la elaboración de harina y bebidas alcohólicas y refrescantes.

El uso prehispánico de Prosopis en Sudamérica está apoyado por numerosos registros arqueobotánicos con una raíz temporal relativamente profunda (Bonomo et al. 2011; Giovanetti et al. 2008). Sin embargo, la diversidad contextual y la amplitud geográfica de dichos registros son aún significativamente menores que las surgidas de las informaciones etnohistóricas. Desde el punto de vista geográfico, todos los registros arqueobotánicos de macrorrestos se ubican al norte del río Colorado -límite norte de la Patagonia- y, en su mayoría, proceden de contextos correspondientes a sociedades con prácticas agrícolas/hortícolas. No se conocían, hasta ahora, datos arqueológicos para el extremo sur del continente, y los únicos obtenidos en contextos vinculados con grupos con economía exclusivamente cazadora-recolectora, corresponden a ocupaciones de los períodos arcaicos y tempranos del noroeste (Capparelli y Lema 2011) y centro oeste de Argentina (Llano 2011). La señal arqueológica más directa de ingesta de estos frutos proviene del norte de Chile donde se registraron restos de vainas en los contenidos intestinales de un cuerpo humano momificado naturalmente (Holden y Núñez 1993).

En este trabajo se dan a conocer los resultados de los análisis efectuados sobre los restos arqueobotánicos de algarrobo (Prosopis spp.), recuperados en un sitio (Angostura 1) del Holoceno tardío del este de Norpatagonia (Argentina). La información presentada constituye un aporte relevante sobre el consumo de los frutos de esta leguminosa en el Cono Sur de América del Sur. En primer lugar, porque son los primeros registros arqueológicos de la explotación alimentaria de este género en Pampa y Patagonia (considerando a ésta en su distribución más tradicional). Y, en segundo lugar, porque provienen de un contexto vinculado con sociedades cazadoras-recolectoras para las que, como fue señalado, se disponía de escasa información. Por otro lado, y teniendo en cuenta que el procesamiento de alimentos es una variable crítica para entender los modos de vida de sociedades pasadas (Capparelli et al. 2011), además del análisis de la muestra arqueológica se efectuaron estudios experimentales a fin de contrastar las hipótesis explicativas del contexto del sitio. Estos estudios se centraron, en primer lugar, en generar indicadores para reconocer, a partir del registro arqueobotánico de macrorrestos, la potencial manufactura de diferentes derivados del algarrobo. En segundo lugar, se reprodujeron los efectos que algunos factores posdeposicionales pudieron haber tenido sobre el registro, a fin de distinguir entre patrones de fragmentación derivados de éstos y aquellos generados por el procesamiento.

Los objetivos de este trabajo son (1) evaluar las evidencias que apoyan la hipótesis de la incorporación antrópica con fines alimentarios de los frutos de algarrobo en el sitio; (2) reconocer los modos posibles de procesamiento y consumo de este recurso en el sitio y (3) discutir el aprovechamiento de estos vegetales en la subsistencia de los grupos del área.

\section{El Área de Estudio}

El sitio Angostura 1 es un sitio a cielo abierto localizado en el este de Norpatagonia (departamento de General Conesa, provincia de Río Negro). Se encuentra en el sector norte del valle del río Negro, a $60 \mathrm{~m}$ de la ribera actual (Figura 2) y a $150 \mathrm{~km}$ de su desembocadura en el océano Atlántico $\left(40^{\circ}\right.$ 
10'19" S y $64^{\circ} 11^{\prime} 22$ "'0"). El río Negro es la principal cuenca fluvial de la Patagonia y discurre por una extensa región árida sin recibir el aporte de ningún tributario. A lo largo de toda la cuenca se ha registrado una gran cantidad de sitios arqueológicos (ca. 250), generalmente asociados con paleolagunas formadas en el interior de palocauces (Prates 2008). La mayor parte de estos sitios, y de las geoformas asociadas, tiene una cronología no mayor a los 3.500 años a.p. y se ha propuesto a partir de ellos un modelo de ocupación basado en el uso de las lagunas y canales de inundación ubicados en la planicie aluvial moderna (Luchsinger 2006; Prates 2008).

El sitio A1 se encuentra en una región climática muy extensa, denominada "diagonal árida", que atraviesa Sudamérica desde el noroeste hasta el sudeste (Iglesias 1981). La temperatura media anual del área de estudio es de $14,2^{\circ}$ y las precipitaciones anuales varían entre 300 (Choele Choel) y 500 mm (proximidades del océano Atlántico) (Frangi y Malacalza 1978). Fitogeográficamente, el área donde está emplazado A1 se ubica en la región Neotropical, Dominio Chaqueño y, si bien Cabrera (1976) la sitúa en la provincia del Monte y Morello (1958:21) en la del Espinal, los dos autores coinciden en que esta zona es un área ecotonal entre ambas. Aunque en la Patagonia, entendida en términos arqueológicos, aparecen principalmente formas arbustivas de Prosopis, como Prosopis denudans Bentham (algarrobillo patagónico), entre otros (Cabrera 1976), también están ocasionalmente presentes formas arbóreas de $P$. flexuosa y $P$. caldenia (Zuloaga et al. 2008). En sectores más meridionales de la Patagonia, la presencia de Prosopis se encuentra circunscrita generalmente a zonas medanosas cercanas a fuentes de agua, y está representada exclusivamente por formas endémicas arbustivas de $P$. denudans (Burkart 1952, Palacios y Brizuela 2005). Aquí las especies del género nunca son dominantes y, según Cabrera (1976), deben considerarse relictos de una antigua dispersión meridional del Monte. En particular, las especies de Prosopis posibles de ser encontradas en los alrededores de A1 son: por un lado, P. caldenia; $P$. alpataco forma alpataco y $P$. flexuosa forma subinermis (propias de la provincia fitogeográfica del Espinal y específicamente del caldenal), por otro lado, P. alpataco forma alpataco, P. flexuosa var. depressa, var. fruticosa, forma flexuosa; P. reptans var. reptans, $P$. strombulifera var. strombulifera

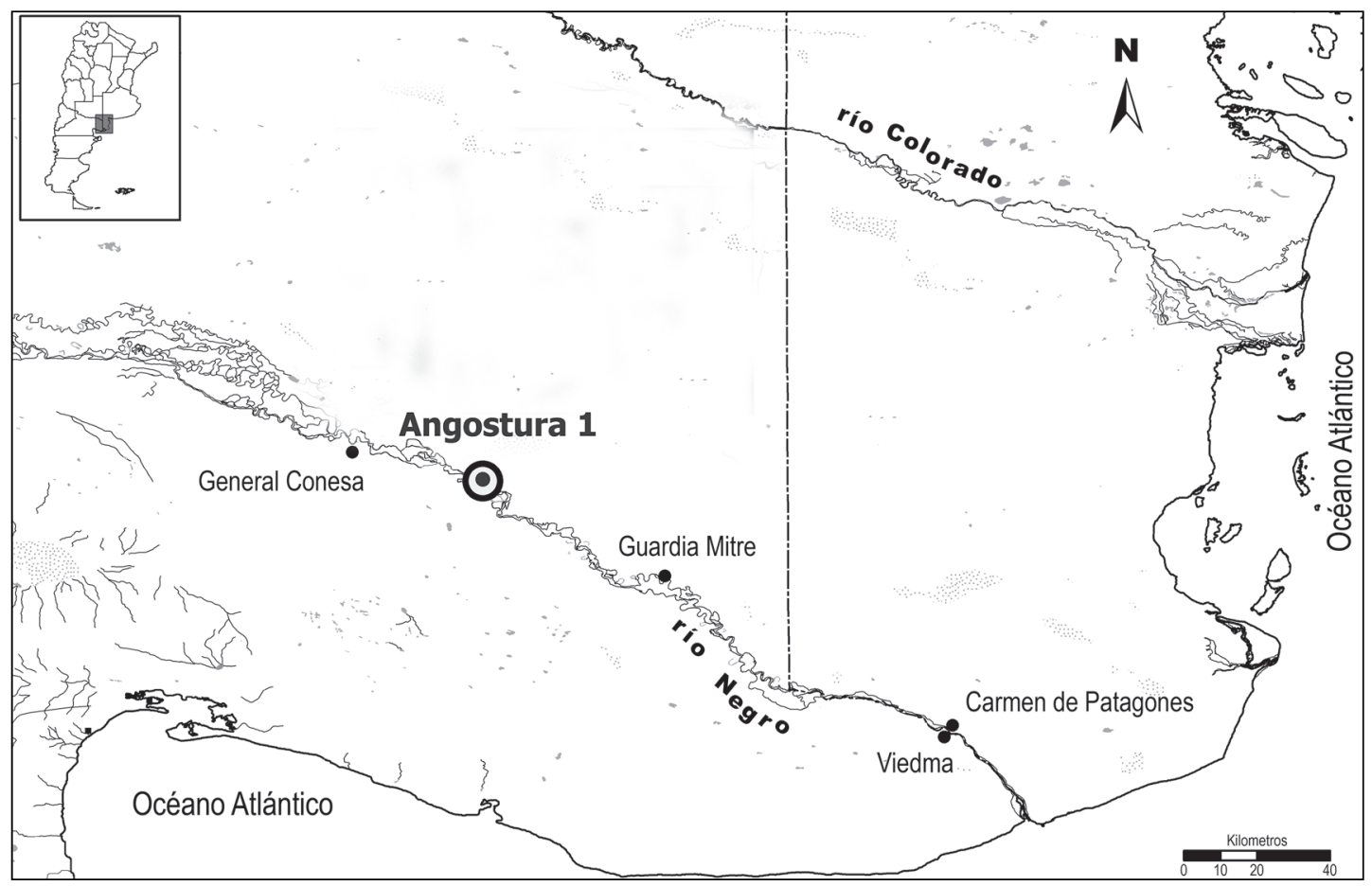

Figura 2. Ubicación del sitio Angostura 1.

Location of the site Angostura 1. 
(propias de la provincia del Monte) y P. denudans var. denudans, var. patagonica (propia de la provincia Patagónica) (Burkart 1952; Cabrera 1976; Morello 1958; Palacios y Brizuela 2005).

\section{Materiales y Método}

Las excavaciones en el sitio A1 se realizaron en una superficie total de $15 \mathrm{~m}^{2}(5 \times 3 \mathrm{~m})$ dividida en unidades menores de un metro cuadrado. La extracción de los sedimentos se llevó a cabo por niveles artificiales de cinco centímetros que se tamizaron con una malla metálica de un milímetro. Se identificaron en el sitio dos componentes arqueológicos, inferior y superior (Unidad 1a y Unidad 3), separados entre sí por sedimentos estériles (Unidad 2). El componente inferior, del que proviene todo el material analizado en este trabajo, fue datado en $938 \pm 45$ años a.p. y, el superior, en $405 \pm 46$ años a.p. (Prates 2008). En el componente inferior los materiales arqueológicos estaban dispuestos alrededor de una estructura de combustión. El tratamiento del material arqueobotánico incluyó dos líneas principales de análisis: la determinación anatómica y taxonómica del material arqueológico, y la realización de estudios experimentales. Como complemento se utilizó la información aportada por cronistas, naturalistas y viajeros sobre los usos y formas de procesamiento de Prosopis por parte de los indígenas de Pampa y Patagonia durante los siglos XVIII y XIX. Todo el material fue escaneado bajo microscopio estereoscópico a fin de aislar los restos de frutos y semillas (o carpológicos), objetos de estudio de este trabajo; otros restos correspondientes a fragmentos de leño o estructuras parenquimatosas no fueron incluidos en este análisis. Los restos carpológicos se identificaron a nivel específico mediante la utilización de muestras comparativas de referencia. Para la experimentación se aplicaron técnicas similares a las empleadas por Capparelli (2008). Se produjeron residuos de molienda de las especies de Prosopis presentes en la zona (a excepción de aquellas con frutos retorcidos, como los de $P$. strombulifera o $P$. reptans, ya que no había evidencia de ellos entre los restos de A1). El tostado y carbonizado experimental de las vainas se realizó en arena caliente (entre $300^{\circ}$ y $355^{\circ} \mathrm{C}$ medidos con termocupla y termómetro digital). El tiempo de exposición al calor varió entre uno y cinco minutos según el caso. La molienda experimental se realizó mediante mortero y mano de piedra.
La fractura post-carbonización se evaluó a través del golpeado suave de las vainas carbonizadas en mortero de vidrio con pilón de vidrio.

\section{Resultados y Discusión}

\section{Descripción de la muestra arqueobotánica}

En A1 se recuperaron 3285 fragmentos vegetales, todos ellos carbonizados. El 97,6\% $(n=3.206)$ corresponde a estructuras leñosas, incluidos fragmentos de cactáceas; algunas, incluso, con remanentes de espinas, y fragmentos de parénquima (en el sentido de Hather 2000). Del 2,4\% restante ( $\mathrm{n}=79$ ), el 97,5\% son partes de vaina de Prosopis $(n=77)$ y el resto corresponde a un endocarpo bilocular de Rhamnaceae y a un fragmento de una hoja de planta suculenta aún no identificada (Tabla 2). Dentro del conjunto de restos de Prosopis $(\mathrm{n}=77)$ predominan los fragmentos de vaina, como artejos enteros y medios artejos (esta categoría corresponde a artejos fragmentados por la línea de sutura de los endocarpos ${ }^{1}$ ) (Figura 3) que en conjunto alcanzan el 37,7\% (Tabla 3). Aunque el 57,2\% corresponde a restos de endocarpos (Figura 3), este valor debe considerarse sobreestimado debido a que solo 9 de ellos están enteros y 35 son fragmentos muy pequeños (Tabla 3). Nótese en la Tabla 3 que los endocarpos fragmentados representan el $0,05 \%$ del peso de la muestra; si esta categoría es omitida en el conteo absoluto, la abundancia de fragmentos de vaina con mesocarpo comestible asciende a $71,7 \%$ y $81,7 \%$ en términos de cantidad y peso, respectivamente (Tabla 3 ). Se recuperó solamente una semilla entera (Figura 3 ) y una cubierta seminal (2,6\%). Los restos de Prosopis fueron identificados en trabajos previos como $P$. denudans, excepto por un endocarpo y cuatro artejos que parecen corresponder a P. alpataco (Capparelli y Prates 2010).

\section{Asociación de la muestra al contexto arqueológico}

En cuanto a la asociación de los restos de Prosopis al contexto del componente inferior del sitio, varios datos sugieren que fueron acumulados por la acción humana y que su incorporación estuvo vinculada con su aprovechamiento alimentario (Prates 2008). Por un lado, la estrecha asociación espacial de las semillas con el resto de los materiales arqueológicos, por otro, los varios antecedentes de 
uso prehispánico de fruto de Prosopis en Argentina, y por último, los registros provenientes de documentos escritos específicos de la Patagonia, que demuestran el uso alimentario del fruto para momentos posthispánicos (véase más abajo).

Aunque, dada la presencia de evidencias de combustión en todos los restos recuperados y su íntima asociación con un contexto de fogón, no puede descartarse totalmente que el algarrobo haya
Tabla 2. Categorías de macrorestos vegetales recuperados del sitio Angostura 1.

Categories of plant macroremains recovered from Angostura 1 site.

\begin{tabular}{rcl}
\hline \multicolumn{1}{c}{ N } & $\%$ & \multicolumn{1}{c}{ Categoría } \\
\hline 3.206 & 97,6 & Madera+Cactaceae?+parenquima \\
77 & 2,28 & Partes de frutos de Prosopis \\
1 & 0,06 & Fragmento hoja suculenta \\
1 & 0,06 & Endocarpo bilocular de Rhamnaceae
\end{tabular}

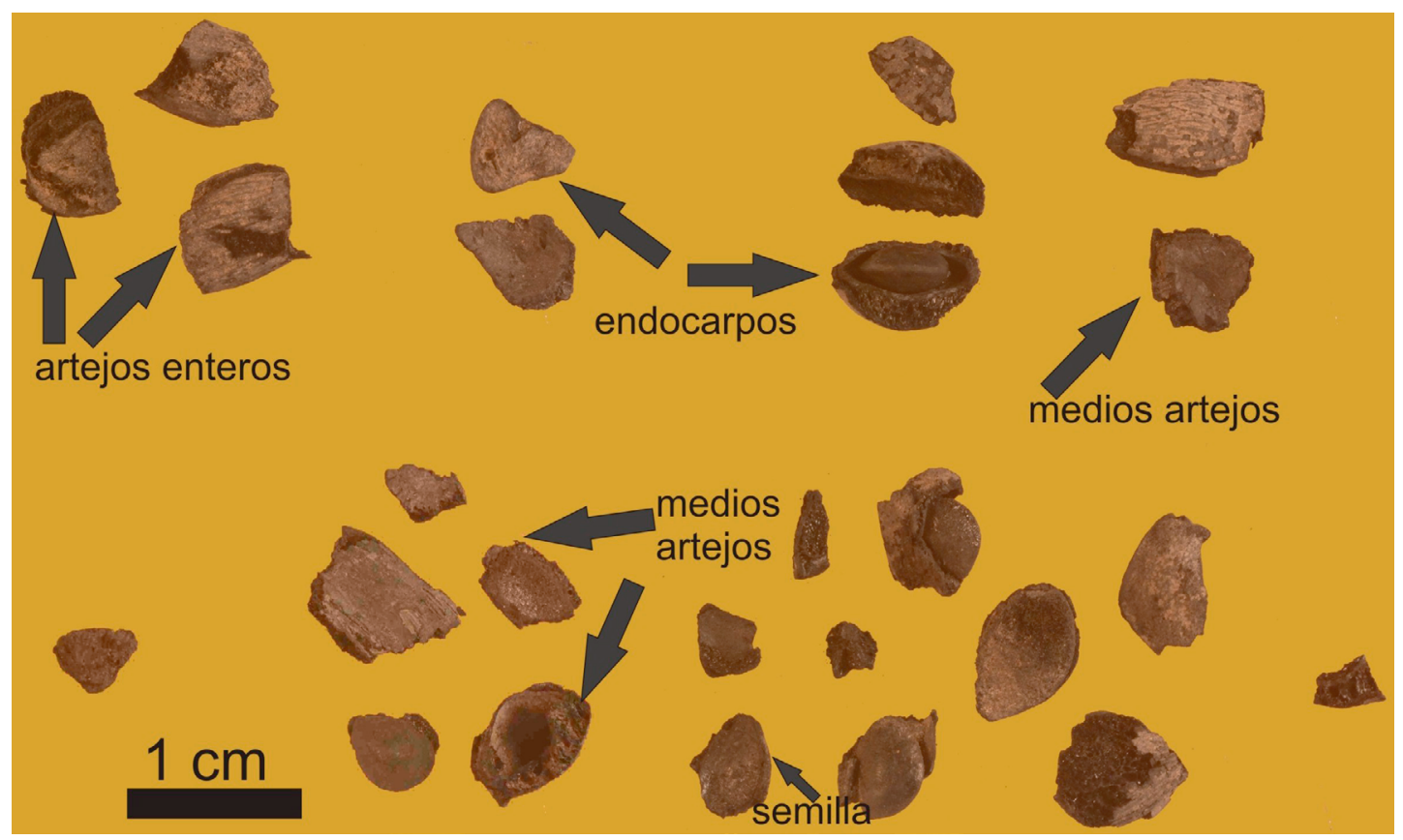

Figura 3. Muestra representativa de los macrorrestos de Prosopis spp. de A1. Unidades estratigráficas: 3b 285-290 cm; 4a 235-240, 245-250 cm; 4b 245-250, 265-270 cm; 5a 285-290, 5b 270-275 cm.

Representative sample of Prosopis spp. plant macroremains from A1. Stratigraphic Units: 36 285-290 cm; 4a 235-240, 245-250 cm; 4 b 245-250, 265-270 cm; 5a 285-290, 5 b 270-275 cm.

Tabla 3. Cantidades absolutas (N) y peso (g) de los macrorrestos de Prosopis spp. del sitio Angostura 1. Absolute frecuencies $(N)$ and weight ( $\mathrm{gr}$ ) of Prosopis spp. macroremains from Angostura 1 site.

\begin{tabular}{|c|c|c|c|c|c|c|}
\hline Categoría & $\mathrm{N}$ & $\%$ & $\%(\mathrm{~S} / \mathrm{EF})$ & Peso (g) & Peso $(\%)$ & $\begin{array}{c}\text { Peso }(\%) \\
\text { (S/EF) }\end{array}$ \\
\hline Fragmentos de vaina con dos medios artejos & 2 & 2,6 & 4,8 & 0,06 & 5,3 & 5,5 \\
\hline Artejos enteros & 17 & 22,1 & 40,5 & 0,66 & 57,9 & 60,6 \\
\hline Medios artejos & 10 & 13 & 23,8 & 0,16 & 14 & 14,7 \\
\hline Endocarpos enteros & 9 & 11,7 & 21,4 & 0,14 & 12,3 & 12,8 \\
\hline Endocarpos fragmentados $(\mathrm{EF})$ & 35 & 45,5 & - & 0,05 & 4,4 & - \\
\hline Fragmentos de epicarpo-mesocarpo & 2 & 2,6 & 4,8 & 0,01 & 0,9 & 0,9 \\
\hline Semillas enteras & 1 & 1,3 & 2,4 & 0,03 & 2,6 & 2,8 \\
\hline Semillas fragmentadas (cubierta seminal) & 1 & 1,3 & 2,4 & 0,03 & 2,6 & 2,8 \\
\hline Total & 77 & 100 & 100 & 1,14 & 100 & 100 \\
\hline
\end{tabular}

Referencias: $\mathrm{S} / \mathrm{EF}=$ sin considerar endocarpos fragmentados. 
sido utilizado como leña en el sitio, la hipótesis de que los restos de fruto aquí tratados provengan de un uso de este tipo fue desestimada debido a que: (a) la presencia de fragmentos de vaina implicaría haber utilizado, para su combustión, las partes terminales de las ramas -donde se encuentran hojas y frutos-, cuyo rendimiento calórico es menor que el que proporcionan porciones no terminales donde predominan partes de tallo solamente; (b) la mayoría de los frutos, para que permanezcan adheridos a las ramas, deben encontrarse en estado inmaduro, lo cual le otorgaría al material propiedades caloríficas y combustibles poco apropiadas y (c) estudios polínicos (Schabitz 2003) muestran que las condiciones climáticas en el área al momento de ocupación del sitio eran similares a las actuales $\mathrm{y}$, por lo tanto, que era abundante la oferta de recursos leñosos (p.ej., Salix humboldtiana-sauce criollo-, Condalia microphyla -piquillín-, Geoffroea decorticans -chañar-e, incluso, Larrea divaricata -jarilla-) con mejores propiedades combustibles que las ramas terminales verdes de Prosopis. También se desestimó que los especímenes provengan del uso de estiércol (de guanaco -L. guanicoe-, ñandú -Rheidae-, mara -Dolichotis patagonu- zorro (Dusicyon sp.) o vizcacha-Lagostomus maximus(Pelliza et al. 1997) como combustible, en cuyo caso se esperaría, al contrario de lo observado, que la mayoría de los frutos no conserven partes fácilmente digeribles como meso y epicarpos (Figura 3) y, en el caso de los herbívoros, que edocarpos y semillas se encuentren altamente fragmentados, tal como se observa en coprolitos actuales (L. Borrelli comunicación personal 2012).

\section{Formas de procesamiento y consumo de los} frutos de Prosopis en Patagonia mencionadas en las fuentes escritas

De las fuentes ethnohistóricas se desprende que durante los siglos posteriores a la conquista española, las vainas de algarrobo se consumían en Pampa y Patagonia de manera generalizada, sobre todo en las regiones con clima semiárido asociadas a las provincias fitogeográficas del Monte y del Espinal. Las formas de aprovechamiento documentadas son diversas y van desde el consumo sin ningún tipo de procesamiento previo hasta la elaboración de subproductos mediante un complejo proceso de producción. El consumo fresco fue observado generalmente cuando los grupos involucrados se encontraban en tránsito y consistía simplemente en la ingesta de las vainas maduras luego de su extracción de la planta (p.ej., Guinnard 1947 [185659]:100, 106; Musters 1997 [1869-1970]:309); incluso, en la provincia de La Pampa era frecuente que los indígenas lleven consigo contenedores con frutos para consumir durante sus viajes (Mansilla 1993 [1870]:393). Cuando Claraz (1988 [1865-66]) dice que "sin procesar o tostadas en ceniza caliente se mastican y se escupen las semillas y vainas", seguramente hace referencia por "vainas" al epicarpo y por "semillas" a los complejos endocarpo-semilla, comúnmente llamados así por algunos pobladores locales (Capparelli 2007). También fue descrito el consumo de las vainas luego de ser hervidas (Claraz (1988 [1865-66]:140).

Los frutos de algarrobo se empleaban asimismo para la elaboración de harina, que luego era utilizada -mezclada con agua- para la preparación del "pan de algarroba" o "torta india" (Falkner 2003 [1774]:94; Musters (1997 [1869-70]:316), o sea, un producto similar al patay (Capparelli 2007). La harina se obtenía machacando entre dos piedras las vainas secas (Bórmida y Casamiquela 1958-59:166) aunque, como observó Claraz (1988 [1865-66]:140), las vainas solían tostarse un instante antes del machacado. La confección de panes o ladrillos con la harina, a fin de acondicionarla para su conservación, fue registrada por Falkner (2003 [1774]:94).

Los frutos de algarrobo también fueron utilizados con frecuencia para elaborar bebidas. Por ejemplo, Musters (1997 [1869-70]) observa que las vainas de algarrobo eran molidas e hidratadas con agua por mujeres. Este tipo de procesamiento es similar al utilizado en el NOA para preparar añapa, bebida refrescante preparada a partir del remojo de las vainas molidas (Capparelli 2007). También se ha reportado la elaboración de una bebida alcohólica: se molían y machacaban las vainas para luego mezclarlas con agua y dejarlas fermentar; de tanto en tanto se quitaba la espuma y se agregaban más vainas hervidas (p.ej., Guinnard 1947 [1856-59]:106); este procesamiento es similar al empleado en la elaboración de la "aloja" en el NOA (Capparelli 2007). Musters (1997 [1869-70]) también menciona el destilado de frutos de algarrobo para la zona de la cordillera.

De todas las observaciones mencionadas en las fuentes escritas, las realizadas en sectores próximos al área de estudio de este trabajo revisten un interés especial, particularmente, las de los viajes de Claraz 
y Musters (trayectoria de viajes en Ciampagna y Capparelli 2012). Ambos autores reportan un amplio rango de formas de procesamiento y consumo de algarrobo, comparable al registrado para el NOA (Capparelli 2007, 2008): (1) consumo de las vainas sin procesar o tostadas en ceniza caliente, (2) elaboración de harina a partir de vainas tostadas, machacadas entre piedras y tamizadas $(\sim$ harina para patay), (3) hervido de vainas, (4) hidratado de vainas molidas ( añapa). Si bien en el NOA el secado de las vainas previo a su procesamiento se realiza comúnmente mediante exposición al sol, en el norte de Patagonia las fuentes etnohistóricas hacen referencia a su tostado en cenizas calientes. Por otra parte, el registro del destilado de los frutos ( aloja) mencionado por Musters, no corresponde al área que nos interesa sino a la localidad de las Manzanas, en el Oeste de Norpatagonia (Ciampagna y Capparelli 2012). Es interesante mencionar aquí lo señalado por Villarino (1972 [1782-83]:94) en cuanto a que los tehuelches no hacían ninguna bebida, a diferencia de los manzaneros que la producían con manzanas y algarroba.

\section{Expectativas arqueológicas sobre los restos de Prosopis de A1 y sus posibles contextos de procesamiento}

Las características de los fragmentos botánicos derivados del consumo directo o de la manufactura de productos intermedios (i.e. harina) y finales (i.e. añapa, patay), así como las de los residuos que las prácticas de procesamiento generan, fueron evaluados en trabajos previos para restos desecados y carbonizados de Prosopis flexuosa y P. chilensis (Capparelli 2008, 2011; Capparelli y Lema 2011). En este trabajo hemos evaluado las correspondientes a P. denudans, P. alpataco y P. caldenia (Tabla 4, Figura 4). La información experimental y los datos etnohistóricos mencionados en el acápite anterior sirvieron de base para definir las expectativas arqueológicas concretas de los modos de consumo y procesamiento de los frutos de Prosopis en A1. La elevada presencia de partes de fruto, es decir, de partes que poseen el mesocarpo interno comestible (Figura 3) en la muestra arqueobotánica de A1 y su asociación con un fogón doméstico, podrían

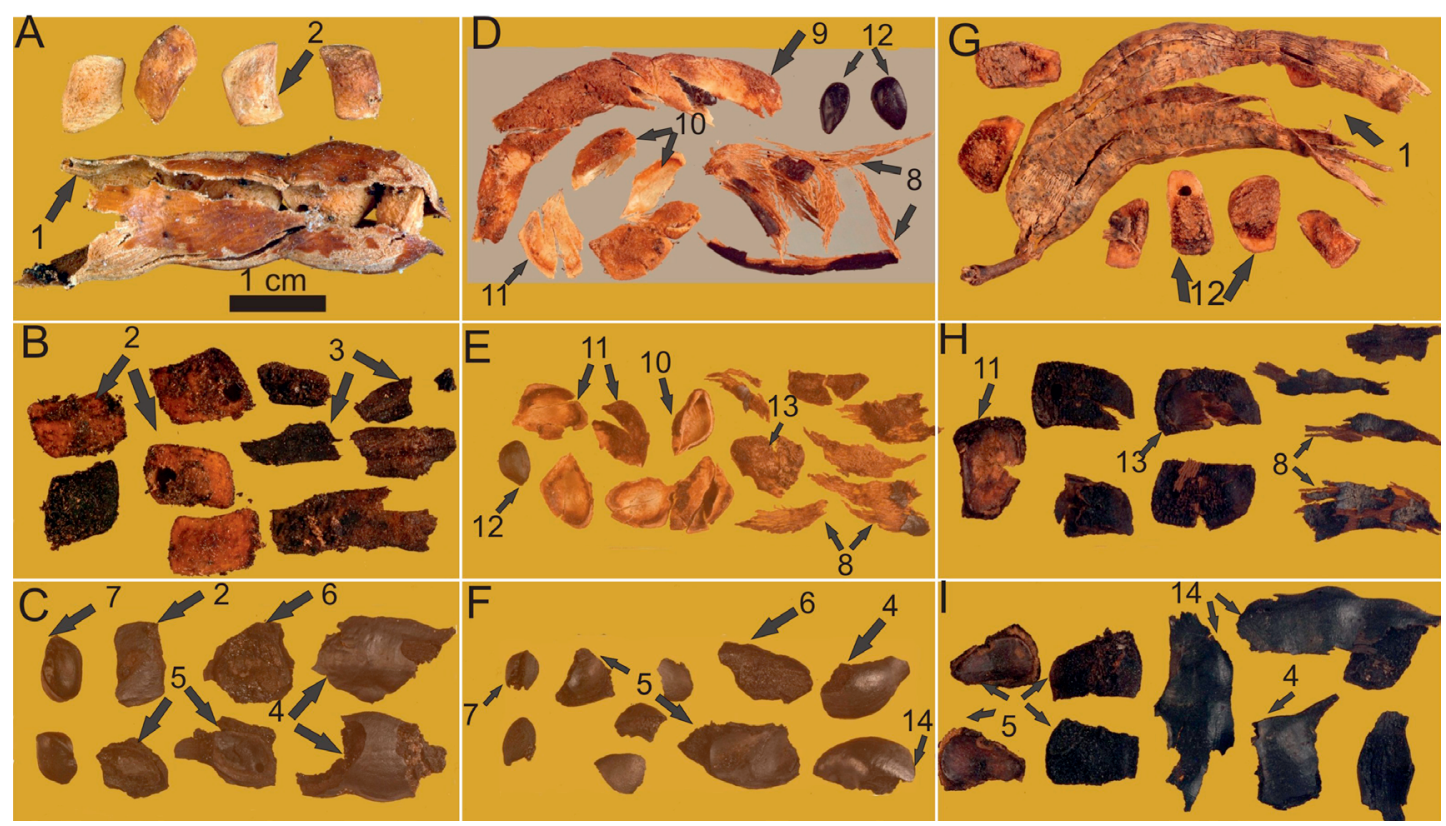

Figura 4. (A-I). Experimentación con Prosopis patagónicos de frutos no retorcidos (los números de los caracteres diagnósticos se corresponden con los mencionados en la Tabla 4). (A-C). P. alpataco. (D-F). P. denudans. (G-I). P. caldenia. (A-H). Molidos en mortero (hasta extraer la harina del mesocarpo interno dulce). (A, D, G). Secados al aire previo a la molienda. (B, E, H). Tostados previo a la molienda. (C, F, I). Carbonizados y golpeados a pilón (para simular procesos posdepositacionales).

(A-I). Experimentation with patagonian Prosopis with untwisted fruits (the numbers of diagnostic features match with those mentioned in Table 4). (A-C). P. alpataco. (D-F). P. denudans. (G-I). P. caldenia. (A-H). Ground with mortar (up extracting the sweet inner mesocarp flour). $(A, D, G)$. Air dried prior to grinding. $(B, E, H)$. Toasted prior to grinding. $(C, F, I)$. Charred and hit with pylon (to simulate postdepositional processes). 


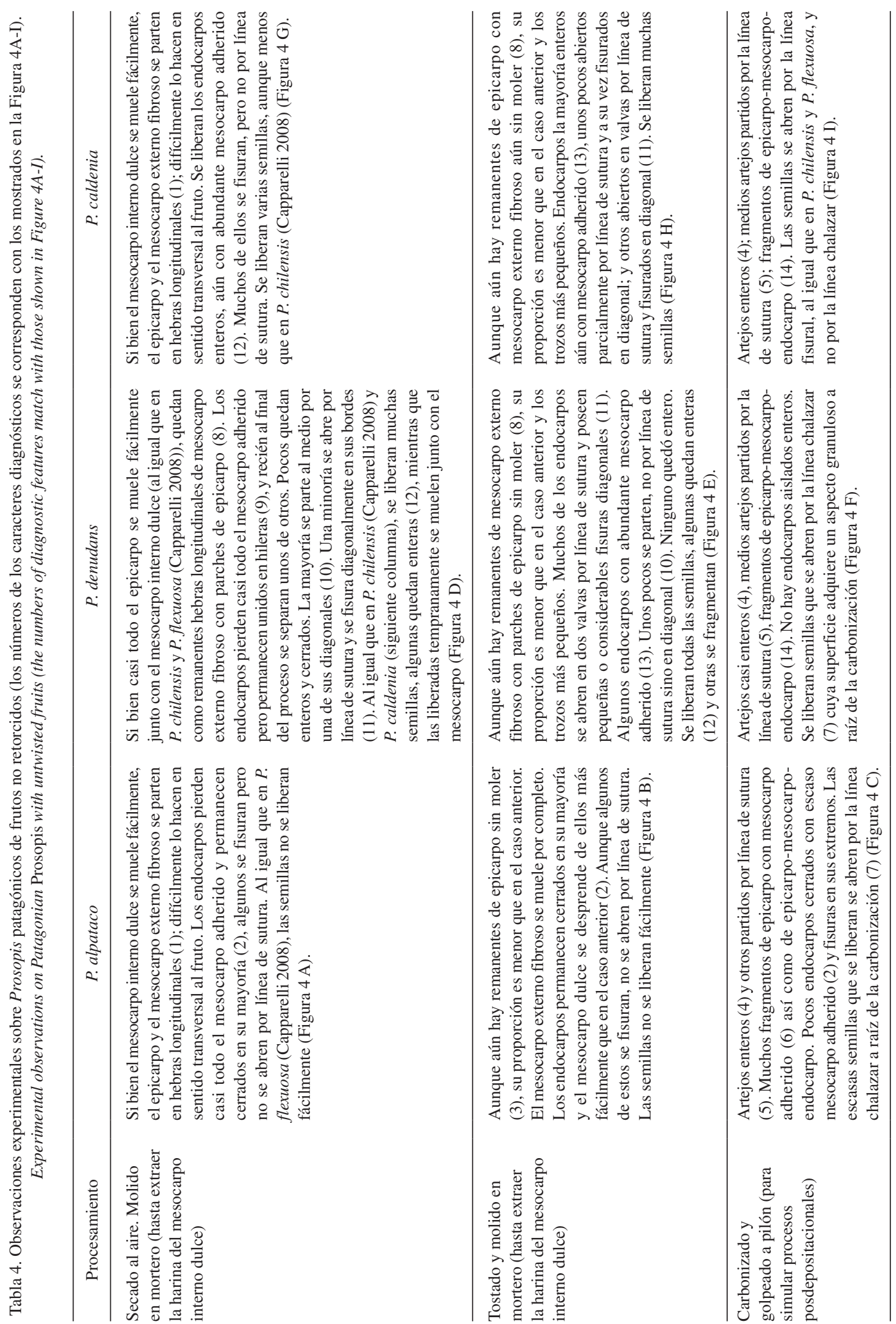


indicar que constituyen una fracción de descarte intencionalmente carbonizada, o bien una fracción que al ser procesada en cercanías y/o mediante el uso del fuego fue accidentalmente carbonizada. Dado que las vainas no presentan razones evidentes para ser desechadas (i.e. orificios de brúquidos) consideramos poco probable la primera hipótesis y, por lo tanto, nos enfocaremos en la segunda, que analizaremos a continuación con mayor profundidad.

Para determinar si la muestra analizada fue o no procesada y las posibles razones de su carbonización, se realizaron los siguientes análisis:

(a) se evaluó si los restos de vaina con mesocarpo comestible presentan evidencias de los procedimientos de remojo en agua fría o hervido mencionados por las fuentes escritas. Como ha sido observado previamente (Capparelli y Lema 2011) ambas acciones generan modificaciones similares en las distintas especies de Prosopis, como disolución de parte del mesocarpo y el plegamiento o enrollamiento del epicarpo en los bordes de los artejos, características que pueden reconocerse incluso en restos carbonizados (Capparelli 2011). Ninguno de estos rasgos diagnósticos fue observado en las partes de vaina recuperadas de A1.

(b) Se evaluó si los restos de Prosopis recuperados en $\mathrm{A} 1$ podrían constituir residuos del consumo directo o de la molienda de vainas para la producción de harina. Capparelli y Lema (2011) observaron que la masticación de los frutos maduros de $P$. chilensis y $P$. flexuosa produce como residuo endocarpos enteros que, en el caso de ser terminales, generalmente van acompañados de restos de epicarpo en forma de finas hebras entrelazadas. Es posible entonces que los escasos restos de endocarpos recuperados en el sitio constituyan el desecho del consumo directo, mediante mascado, de vainas tostadas previamente. Aunque resulta llamativa la ausencia de hebras entrelazadas de epicarpo, esto podría deberse a su poca resistencia al proceso de carbonización (Capparelli 2011).

La experimentación del mascado y molido de las vainas de $P$. denudans, $P$. alpataco y $P$. caldenia permitió observar que poseen cubiertas (i.e. suma de epicarpo y mesocarpo externo fibroso) de consistencia más gruesa y fibrosa que las de $P$. flexuosa y $P$. chilensis, siendo las de $P$. alpataco las más gruesas de todas. Esta característica no solo dificulta su digestibilidad, sino también la molienda de las vainas secas, ya que produce grandes fragmentos de epicarpo y/o mesocarpo externo fibroso, aún luego de extraer el mesocarpo dulce (Tabla 4 fila 1, Figura 4a, d, g). Esta situación es diferente a la observada en trabajos previos para $P$. chilensis y P. flexuosa (Capparelli 2008), donde el secado al sol de las vainas es suficiente para que el epicarpo y el mesocarpo externo se pulvericen junto con el mesocarpo interno durante la molienda.

(c) Se observó también que el tostado previo de las vainas de las especies patagónicas hace que las cubiertas gruesas se mastiquen o muelan más fácilmente (Tabla 4 fila 2, Figura 4b, e, h). En $P$. caldenia (Figura 4h) y P. alpataco (Figura 4b) los endocarpos suelen permanecer enteros luego de la molienda de las vainas tostadas, mientras que en P. denudans (Figura 4e) es común que los endocarpos se abran por la línea de sutura o se fracturen diagonalmente después de extraído el mesocarpo. Aunque este último rasgo es difícil de evaluar en restos carbonizados porque son muy susceptibles a la fragmentación, su ausencia en la muestra de A1 -en su mayoría correspondiente a P. denudans (Capparelli y Prates 2010)- sugiere que las vainas no habrían sido aún molidas. Esto es congruente también con el hecho de que en A1 hay preponderancia de partes de vaina que aún poseen gran cantidad de mesocarpo interno dulce (artejos y medios artejos) (Figura 3), las que no son predominantes en las muestras experimentales tostadas y molidas (Figura $4 \mathrm{~b}, \mathrm{e}, \mathrm{h}$ ).

(d) Por último, se comparó el patrón de fragmentación registrado en las partes de vaina del sitio con el patrón de fractura experimental observado en vainas carbonizadas y posteriormente golpeadas (Capparelli 2011) (Tabla 4 fila 3, Figura $4 \mathrm{c}, \mathrm{f}, \mathrm{i})$. Esto permitió determinar que el patrón de fragmentación de los restos de Prosopis ssp. de A1 se habría producido con posterioridad a la carbonización y, posiblemente, como consecuencia de la acción de agentes posdepositacionales.

En suma, si bien el consumo directo del fruto de Prosopis no puede ser totalmente descartado en A1, el predominio de fragmentos de vainas con partes comestibles, artejos enteros y medios artejos; su asociación a un contexto de fogón donde otros productos alimentarios estaban siendo procesados y/o consumidos (moluscos, mamíferos y peces) (Fernández et al . 2011; Prates y Marsans 2007); la mención en las fuentes etnohistóricas de Patagonia de la práctica de tostar las vainas antes de su consumo directo o de su molido 
para la manufactura de harina; y los resultados de la experimentación permiten sostener que la mayoría de los restos provendría de vainas que se carbonizaron accidentalmente mientras eran tostadas en cenizas calientes.

La práctica del tostado es un tipo de procesamiento poscosecha (sensu Capparelli y Lema 2010) que en este caso vincula los frutos de Prosopis al área residencial -y más específicamente al fogón-, incide en su potencial carbonización, e implica un aumento de las condiciones de preservación/ visibilidad arqueológica de los restos vegetales. En contraste, el consumo directo de frutos silvestres ( $\sin$ procesamiento previo) y fuera de las áreas residenciales, durante el traslado de las personas de un lugar a otro, posee baja visibilidad arqueológica a nivel de macrorrestos botánicos. Esta podría ser una causa razonable de la ausencia de registros previos de Prosopis en otros sitios de Patagonia, más aun considerando el predominio de condiciones favorables para su preservación (p.ej., ambientes áridos y de suelos aridisoles), incluso de aquellos en estado seco. Cabe aclarar que consideramos improbable que la ausencia de registros previos de vainas de Prosopis y su aparición ca. 900 años a.p. puedan deberse a la ausencia del género en las comunidades vegetales del área en momentos previos a la ocupación del sitio y a una expansión posterior motivada por acciones humanas (i.e. desertificación o introducción de ganado). Esta consideración se soporta en primer lugar, porque la invasión de formas arbustivas de Prosopis (que se comportan como maleza) se asocia frecuentemente a la deforestación de las formas arbóreas y al desarrollo de la agricultura (i.e. Beresford Jones 2011; Paziecznik et al. 2001), acciones para las cuales no existen evidencias en el área (ver por ejemplo Prates 2008). En segundo lugar, porque en los momentos de ocupación de Angostura I el rango de dispersión del género debió estar controlado, entre otros, por factores ligados a su coevolución con los camélidos (p.ej., Mooney et al. 1977). Y en tercer lugar, porque varios estudios antracológicos prueban la presencia de Prosopis y el uso de partes leñosas (Perez de Micou 1995) en momentos previos en otros sectores más meridionales de Patagonia. Por ejemplo, fueron registrados carbones de madera de Prosopis denudans en Chubut datados entre 4470 y 1750 años a.p. (Marconetto 2002) y de Prosopis sp. en la costa norte de Santa Cruz el (Ciampagna 2014a) desde los 1690 años a.p. (sitio Alero 4,
Ambrústolo y Ciampagna 2014) hasta los 690 años AP (sitios La Estrella y Palo Alto, Ciampagna 2014a) e incluso en sitios poscontacto (Nido del Águila, Ciampagna 2014b).

Volviendo entonces a la idea que sostenemos en el presente trabajo, de que los frutos de Prosopis se hacen visibles en forma de macrorrestos en el registro de Angostura I a partir de un cambio en los modos de procesamiento (tostado de las vainas previo a su consumo), lo que facilita su ingreso a la esfera doméstica del sitio, vale la pena mencionar que otras prácticas poscosecha (molido, por ejemplo) pueden invisibilizar las evidencias en forma de macrorrestos en el registro arqueológico a favor de aquellas del tipo de microrrestos. A diferencia de lo ocurrido en $\mathrm{A} 1$ donde hasta el momento no se han recuperado artefactos de molienda, datos procedentes de sitios del noroeste (Aquihuecó y Michacheo) y sur (Punta Medanosa) de Patagonia, en los que no se recuperaron macrorrestos botánicos del género, validan la ocurrencia de prácticas de molienda en el área. Nos referimos a los estudios realizados recientemente sobre instrumentos de molienda en los que se observaron granos de almidón de Prosopis (tanto regulares como irregulares de tipo B2, altamente refringentes y de hilio fusiforme) (Lema et al. 2012) y corpúsculos de resina que podrían asociarse al mismo género (Ciampagna et al. 2012). Es interesante destacar entonces la importancia de aplicar todas las vías metodológicas posibles, no solo de macro, sino de microrrestos vegetales, para evaluar el uso alimentario de las vainas de Prosopis en la región, que, en coincidencia con lo propuesto también por Moreno y Escobar (2001) y Gómez Otero (2007), parece ser más importante de lo que hasta ahora ha demostrado su registro arqueológico.

\section{Conclusiones}

El registro etnohistórico de Patagonia muestra que los frutos de Prosopis se consumían de diversas maneras desde momentos poshispánicos más o menos tempranos (i.e. Flakner 1774). Sin embargo, eran muy escasas hasta ahora las evidencias para tiempos previos. Los materiales analizados en este trabajo constituyen los primeros registros de macrorrestos para el área. La evidencia presentada permite señalar que en este sitio los frutos habrían sido destinados al consumo y que la mayoría corresponde a elementos enteros fragmentados 
con posterioridad a su depositación. Las vainas se habrían carbonizado por accidente mientras eran tostadas en cenizas calientes, probablemente como una etapa previa al molido. Esta práctica de tostado determinó la asociación de los frutos de Prosopis al área residencial -y más específicamente al fogón-, y ofreció - por medio de la carbonización- las condiciones para su preservación y visibilidad arqueológicas.
Agradecimientos: A Bruno Pianzola por colaborar con la toma de fotografías a los especímenes de Prosopis y a la Secretaría de Cultura de la provincia de Río Negro por proveer los permisos para realizar los trabajos de campo. A los revisores anónimos por sus sugerencias. Este trabajo fue realizado gracias al financiamiento de la ANPCyT (PICT-2012-0242 titular Gustavo Martínez) y del CONICET (PIP 0459 titular Aylen Capparelli).

\section{Referencias Citadas}

Ambrústolo, P. y M.L. Ciampagna 2014. Study of Alero 4 rock shelter, North coast of Deseado Estuary (Patagonia, Argentina). Quaternary International, en prensa.

Beresford-Jones, D.G. 2011. The Lost Woodlands of Ancient Nasca. A Case-study in Ecological and Cultural Collapse. Oxford University Press, Oxford.

Bonomo, M., F.J. Aceituno, G.G. Politis y M.L. Pochettino 2011. Pre-Hispanic horticulture in the Paraná Delta (Argentina): archaeological and historical evidence. World Archaeology 43:554-575.

Bórmida, M. y R.M. Casamiquela 1958-59. Etnografía gününakëna. Testimonio del último de los tehuelches septentrionales. Runa 9 (N.S) (1-2):153-193.

Burkart, A. 1940. Materiales para una monografía del género Prosopis (Leguminosae). Darwiniana 4:57-128

Burkart, A. 1952. Las Leguminosas Argentinas Silvestres y Cultivadas, segunda edición. Acme, Buenos Aires.

Burkart, A. 1976. A monograph of the genus Prosopis (Leguminosae subfam. Mimosoideae). Journal of the Arnold Arboretum 57:217-525.

Cabrera, A.L. 1976. Regiones fitogeográficas argentinas. En Enciclopedia Argentina de Agricultura y Jardinería, tomo II, fascículo 1. Acme, Buenos Aires.

Capparelli, A. 2007. El Algarrobo blanco y negro -Prosopis chilensis (Mol.) Stuntz y P. flexuosa DC, Fabaceae- en la vida cotidiana de los habitantes del NOA: subproductos alimenticios. Kurtziana 33:103-119.

Capparelli A. 2008. Caracterización cuantitativa de productos intermedios y residuos derivados de alimentos del algarrobo (Prosopis flexuosa DC y P. chilensis (Mol.) Stuntz, Fabaceae): aproximación experimental aplicada a restos arqueobotánicos desecados. Darwiniana 46:175-201.

Capparelli, A. 2011. Elucidating post-harvest practices involved in the processing of algarrobo (Prosopis spp.) for food at El Shincal Inka site (Northwest Argentina): an experimental approach based on charred remains. Journal of Archaeological and Anthropological Sciences 3:93-112.

Capparelli, A. y V. Lema 2010. Prácticas "post-aprovisionamiento" de recursos vegetales: una perspectiva paleoetnobotánica integradora aplicable al desarrollo de la arqueobotánica argentina. En Arqueología Argentina en el Bicentenario de la Revolución de Mayo, editado por R. Bárcena y H. Chiavazza, pp. 1171-1176. Universidad Nacional de Cuyo, Mendoza.

Capparelli, A. y V. Lema 2011. Recognition of post-harvest processing of algarrobo (Prosopis spp.) as food from two sites of Northwestern Argentina: an ethnobotanical and experimental approach for desiccated macroremains. Journal of Archaeological and Anthropological Sciences 3:71-92.

Capparelli, A y L. Prates 2010. Identificación específica de frutos de algarrobo (Prosopis spp., Fabaceae) y mistol (Ziziphus mistol Griseb. Rhamnaceae) en un sitio arqueológico de Patagonia. En Traditions and Transformations in Ethnobotany, editado por M.L. Pochettino y A. Ladio, pp. 13-19. Cyted, San Salvador de Jujuy.

Capparelli, A., S. Valamoti y M. Wollsonecroft 2011. After the Harvest: investigating the role of food processing in past human societies. Archaeological and Anthropological Sciences 3:1-5.

Ciampagna, M.L. 2014a. Estudio de la Interacción de los Grupos Cazadores Recolectores que Habitaron la Costa Norte de Santa Cruz y las Plantas Silvestres: Recolección y Gestión. Tesis doctoral presentada para ser evaluada en la Facultad de Ciencias Naturales y Museo, Universidad Nacional de La Plata, La Plata.

Ciampagna, M.L. 2014b. Estudio antracológico de los sitios Cormorán Quemado y Nido del Águila, Costa Norte de Santa Cruz, Argentina. Ponencia presentada en IX Jornadas de Arqueología de Patagonia, Coyhaique.

Ciampagna, M.L. y A. Capparelli 2012. Historia del uso de las plantas por parte de las poblaciones que habitaron la Patagonia Continental argentina. Cazadores Recolectores del Cono Sur. Revista de Arqueología 6:45-75.

Ciampagna L., V. Trola, L. Borrelli y A. Capparelli 2011. El uso de recursos vegetales por parte de grupos cazadores recolectores de la costa norte de Santa Cruz: análisis de instrumentos de molienda. Ponencia presentada en VIII Jornadas de Arqueología de la Patagonia, Malargüe.

Claraz, J. 1988 [1865-66]. Diario de viaje de Exploración al Chubut. Marymar, Buenos Aires.

Falkner, T. 1911 [1774]. Descripción de la Patagonia. Coni, Buenos Aires.

Felker, P. 1979. Mesquite, an all purpose leguminous arid land tree. En New Agricultural Crops, editado por G.A. Ritchie, pp. 89-132. Westview Press, Colorado. 
Fernández Distel, A. 1989. Una nueva cueva con maíz acerámico en N.O. argentino: León Huasi 1, excavación. Revista Comunicaciones Científicas 1:4-17.

Fernández, F., L. Del Papa, G. Moreira, L. Prates y L. De Santis 2011. Small mammal remains recovered from two archaeological sites in the middle and lower Negro river valley (Late Holocene, Argentina). Taphonomic issues and paleoenvironmental implications. Quaternary International 245:136-147.

Frangi, J.L. y L. Malacalza 1978. Estudio económico para el desarrollo integral del valle del río Negro: informe ecológico de consultoría para el Ing. Conrado Bauer. Manuscrito en posesión de los autores.

Gómez Otero, J. 2007. Dieta, Uso del Espacio y Evolución en Poblaciones Cazadoras-Recolectoras de la Costa CentroSeptentrional de Patagonia Durante el Holoceno Medio y Tardío. Tesis para optar al grado de Doctora en Filosofía y Letras, Universidad de Buenos Aires, Buenos Aires.

Guinnard, A. 1947 [1856-59]. Tres Años de Esclavitud entre los Patagones; Relato de mi Cautiverio. Espasa Calpe, Buenos Aires.

Holden, T.G. y L. Núñez 1993. An analysis of the gut contents of five well-preserved human bodies from Tarapacá, Northern Chile. Journal of Archaeological Science 20:595-611.

Horkheimer, H. 2004. Alimentación y Obtención de Alimentos en el Perú Prehispánico, Segunda Edición. Instituto Nacional de Cultura, Lima.

Iglesias, A. 1981. Temperaturas. En Atlas Total de la República Argentina, editado por E. Chiozza y R. Figueira, pp. 204-208. Centro Editor de América Latina, Buenos Aires.

Latcham, R. 1936. La Agricultura Precolombina en Chile y los Países Vecinos. Universidad de Chile, Santiago.

Lema, V., A. Capparelli y A. Martínez 2012. Las vías del algarrobo: antiguas preparaciones culinarias en el noroeste argentino. En Las Manos en la Masa: Arqueologías y Antropologías de la Alimentación en Suramérica, editado por M.P. Babot, M. Marschoff y F. Pazzarelli, pp. 639-665. Museo de Antropología de Córdoba, Córdoba.

Llano, C. 2011. Aprovechamiento de los Recursos Vegetales entre las Sociedades Cazadoras-Recolectoras del Sur de Mendoza. Tesis para optar al grado de Doctora en Biología, Universidad Nacional del COMAHUE, Bariloche.

Luchsinger, H. 2006. The Late Quaternary Landscape History of the Middle Rio Negro Valley, Northern Patagonia, Argentina: Its Impact on Preservation of the Archaeological Record and Influence on Late Holocene Human Settlement Patterns. Doctoral Dissertation, A\&M Texas University, Texas.

Marconetto, M.B. 2002. Análisis de vestigios de combustión de los sitios Alero don Santiago y Campo Moncada. En Plantas y Cazadores en Patagonia, compilado por C. Pérez de Micou, pp. 33-54. Facultad de Filosofía y Letras, UBA, Buenos Aires.

Mansilla, L. V. 1993 [1870]. Una Excursión a los Indios Ranqueles. Espasa Calpe, Buenos Aires.
Mc. Laren, F. y A. Capparelli 2004. The early use of Prosopis L. Species as food: Old World and New World evidence. Ponencia presentada en Ninth International Congress of Ethnobiology, Kent.

Mooney H.A., B.B. Simpson y O.T. Solbrig 1977. Phenology, Morphology, Physiology. En Mezquite. Its biology in two desert ecosystems, editado por B.B. Simpson, pp. 26-43. US/ IBP Synthesis Series 4, Dowden, Hutchington \& Ross, Inc., Stroudsburg, Pennsylvania.

Morello, J. 1958. La Provincia fitogeográfica del Monte. Opera Lilloana 2:1-155.

Moreno J.E. y F. Escobar 2001. Algarrobos, artefactos de molienda y cerámica en Patagonia centro sur: la distribución y el uso. Ponencia presentada en XIV Congreso Nacional de Arqueología Argentina, Rosario.

Musters, G.C. 1997 [1869-1970]. Vida entre los Patagones. El Elefante Blanco, Buenos Aires.

Outes, F.F. 1917. Observaciones etnográficas de Francisco Javier Muñiz. Physis 1:197-215.

Palacios, R. y M.M. Brizuela 2005. Fabaceae, parte 13. Subfam. II. Mimosoidea, parte 4. Tribu VI. Mimoseae, parte B. Prosopis L. En Flora Fanerogámica Argentina 92:3-25. IMBIV (CONICET), Programa PROFLORA, Córdoba.

Pasienczik, N.M., P. Felker, P. Harris, L.N. Harsh, G. Cruz, J.C. Tewari, K. Cadoret y L.J. Maldonado 2001. The Prosopis juliflora-Prosopis pallida Complex: A Monograph. Henry Doubleday Research Association (HIDRA), Coventry.

Pérez de Micou, C. 1995. El registro arqueológico como indicador de cambio ambiental. El caso de los macrovestigios vegetales en sitios de la Patagonia Argentina. Cuadernos Instituto Nacional de Antropología 40:177-186.

Prates, L. 2008. Los Indígenas del Río Negro: Un Enfoque Arqueológico. Sociedad Argentina de Antropología, Buenos Aires.

Prates, L. y N. Marsans 2007. El uso de moluscos de agua dulce (Diplodon chilensis patagonicus) en el sitio Angostura 1 (departamento de General Conesa, Río Negro). Intersecciones en Antropología 8:355-359.

Pelliza A., P. Willems, V. Nakamatsu y A. Manro 1997. Atlas Dietario de Herbívoros Patagónicos, editado por R. Somlo. Prodesar - INTA-GTZ, Bariloche.

Villagrán, C., V. Castro, G. Sánchez, M. Romo, C. Latorre y L. Hinojosa 1998. La tradición surandina del desierto: Etnobotánica del área del Salar de Atacama (Provincia de El Loa, Región de Antofagasta, Chile). Estudios Atacameños 16:7-105.

Villarino, D.B. 1972 [1782-83]. Diario del Piloto de la Real Armada D. Basilio Villarino del reconocimiento que hizo del río Negro en la costa oriental de la Patagonia. En Colección Pedro de Angelis, tomo VIII, vol. B, pp. 967-1138. Plus Ultra, Buenos Aires.

Zuloaga, F., O. Morrone y M. Belgrano (eds.) 2008. Catálogo de las Plantas Vasculares del Cono Sur. Disponible en: http://www. darwin.edu.ar/Proyectos/FloraArgentina/Generos.asp?Letra=P, [Consultado el 4 de febrero de 2015]. 


\section{Nota}

1 La consideración de esta nueva categoría y una revisión más detallada de los restos en el presente trabajo modificaron levemente los valores parciales preliminares publicados en Prates 2008:221. 
